\title{
Ortho距过
}

Vol. 16, Núm. 4

Oct.-Dic. 2020. pp 201-211

doi: $10.35366 / 97819$

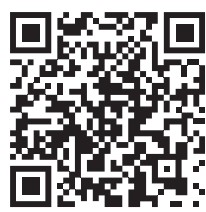

\section{Secuelas de lesión de plexo braquial de nacimiento en hombro}

\author{
Sequels of brachial plexus birth injury in the shoulder
}

\author{
Ana Fernanda Espinosa de los Monteros Kelley, ${ }^{*}$ \\ Jorge Francisco Clifton Correa, ${ }^{\ddagger}$ Rafael Reynoso Campo, ${ }^{\S}$ \\ José Abraham Delgadillo Vargas,"
}

Renata Leal Farias," Leonardo López Almejo, ${ }^{\star \star}$ René Humberto Barraza Arrambide, ${ }^{\ddagger}$ Rogelio Solano Pérez, $\$ \S$ Raúl Emérico Rodríguez Martínez, Eduardo Pablo Zancolli***

\footnotetext{
* Cirujano Ortopedista, Cirujano de Mano, Cirugía de Nervio Periférico, Staff del Hospital Real San José, Guadalajara. Profesor del Diplomado de Cirugía de Plexo Braquial, Universidad Autónoma de Coahuila (UAdeC), Torreón, Coahuila. Máster en Brachial Plexus International School (BPIS).

‡ Cirujano Plástico, Profesor de Cirugía de Plexo Braquial y Nervio Periférico, Hospital Universitario,

UAdeC, Torreón, Coahuila. Profesor del Máster en Cirugía de Plexo Braquial, BPIS.

$\S$ Cirujano Plástico, Cirujano de Mano y Microcirugía, Hospital Ángeles,

Profesor de Cirugía de Plexo Braquial y Nervio Periférico.

^ Cirujano Ortopedista Pediatra, Diplomado de Cirugía de Plexo Braquial y Nervio Periférico de la UAdeC, Torreón, Coahuila.

" Cirujano Ortopedista, Cirujano de Mano, Microcirugía, Diplomado de Cirugía de

Plexo Braquial y Nervio Periférico de la UAdeC. Recife, Brasil.

** Cirujano Ortopedista, Cirujano de Mano, Cirugía de Nervio Periférico,

Staff del Star Médica Aguascalientes, Profesor del Diplomado de Cirugía de

Plexo Braquial de la UAdeC, Torreón, Coahuila. Máster en BPIS.

¥ Cirujano Ortopedista, Cirujano de Mano, Cirugía de Nervio Periférico,

Staff del Christus Muguerza Hospital Saltillo, Profesor.

$\S$ Cirujano Ortopedista, Cirujano de Columna, Cirugía de Nervio Periférico, Staff del

Hospital «Dr. Victorio de la Fuente Narváez», Ciudad de México. Diplomado de Cirugía de Plexo Braquial de la UAdeC, Torreón, Coahuila. Máster en BPIS.

If Cirujano Ortopedista, Cirujano de Mano, Cirugía de Nervio Periférico, Staff del Hospital Puerta de Hierro, Guadalajara. Diplomado de Cirugía de Plexo Braquial de la UAdeC, Torreón, Coahuila. Máster en BPIS.

*** Cirujano Ortopedista, Cirujano de Mano, Cirugía de Nervio Periférico, Staff del Hospital Fundación Favaloro, Buenos Aires, Argentina. Diplomado de Cirugía de

Plexo Braquial de la UAdeC, Torreón, Coahuila. Máster en BPIS.

Correspondencia: René Humberto Barraza Arrambide. Christus Muguerza Hospital Saltillo, Carretera Saltillo-Monterrey Km 4.5, interior 162, 25210, Saltillo,

Coahuila, México. Correo electrónico: cirugiademano.saltillo@gmail.com
}

Citar como: Espinosa de los Monteros KAF, Clifton CJF, Reynoso CR, Delgadillo VJA, Leal FR, López AL y cols. Secuelas de lesión de plexo braquial de nacimiento en hombro. Ortho-tips. 2020; 16 (4): 201-211. https://dx.doi.org/10.35366/97819 


\section{RESUMEN}

Las lesiones de plexo braquial ocurren a pesar de los avances actuales de los cuidados prenatales y obstétricos. La mayoría de los pacientes pueden recuperarse completamente, sin embargo, a pesar de una adecuada reconstrucción nerviosa algunos desarrollan un déficit establecido con secuelas en la articulación del hombro secundarias a un desbalance muscular entre los músculos paralizados y los funcionales. En estos pacientes es obligatoria la evaluación detallada y específica y el tratamiento para mejorar la función, debe enfocarse también en evitar preventivamente una progresión.

Palabras clave: Secuelas, plexo braquial, Lesión del plexo braquial, parálisis del plexo, lesión perinatal, displasia glenohumeral, lesión obstétrica del plexo braquial.

\section{ABSTRACT}

Brachial plexus injuries occur despite current advances in prenatal and obstetric care. Most patients can recover completely; however, despite adequate nerve reconstruction some develop an established deficit with sequelae in the shoulder joint secondary to a muscle imbalance between paralyzed and functional muscles. In these patients, a detailed and specific evaluation is mandatory, and treatment to improve function must also focus on preventing progression.

Keywords: Sequelae, brachial plexus, brachial plexus injury, plexus paralysis, perinatal injury, glenohumeral dysplasia, obstetric brachial plexus injury.

\section{INTRODUCCIÓN}

La incidencia mundial reportada de la parálisis del plexo braquial de nacimiento es de 0.5 a 3 por cada 1,000 nacidos vivos. ${ }^{1-3}$ México tiene una incidencia de 0.37-0.87 por cada 1,000 nacimientos. ${ }^{4}$ La evolución natural de la lesión es hacia cierto grado de mejoría; sin embargo, se ha reportado hasta $33 \%$ de pacientes que presentan deformidad ósea, e incluso hasta $56 \%$ de prevalencia de la contractura en rotación interna, aun con una reinervación casi completa. ${ }^{5}$

Al revisar los casos de lesión de plexo braquial de nacimiento sin tratamiento quirúrgico previo y también a quienes recibieron los modernos estándares de reinervación motora, encontramos un porcentaje muy elevado de pacientes que desarrollan discapacidad permanente, siendo el hombro la articulación más comúnmente afectada. ${ }^{6,7}$

Deducimos que las secuelas se desarrollan en el primer grupo por una recuperación motora incompleta y en el segundo por una reinervación insuficiente después de la adecuada reconstrucción nerviosa.

En cualquiera de los dos grupos, el desequilibrio entre la musculatura no funcional y las fuerzas de deformación consecuentemente anormales por el uso de la musculatura bien inervada causan un impacto permanente en la dinámica de las articulaciones afectadas, deformándolas.

\section{HISTORIA}

Royal Whitman describió en 1900 la luxación de hombro no congénita secundaria a una lesión ocurrida durante el parto y la consecuente contractura en rotación interna de la extremidad. Como sus secuelas asoció una extremidad más corta 
y un hombro anquilosado. Ya desde entonces resaltaba la importancia tanto de la movilización pasiva para prevenirlo como inmovilización durante un tiempo suficiente para lograr el reajuste de los tejidos. ${ }^{8}$

Para el problema, Fairbanks prescribió en 1913 el uso de ortesis y recomendó la liberación capsular anterior con sección del músculo subescapular y la cápsula articular en los casos refractarios. ${ }^{9}$

Posteriormente Sever en 1925 analizó 1,100 casos y describió las alteraciones radiográficas presentadas en estos pacientes, categorizadas como: 1) disminución del tamaño de la cabeza humeral, 2) aplanamiento de la glenoides, 3) subluxación posterior, 4) acromion en forma de gancho y 5) elongación de la apófisis coracoides. Su técnica quirúrgica para corregirlo consistía en la división del pectoral mayor, la resección de la punta del coracoides con división del tendón conjunto y la división completa del subescapular sin capsulotomía. Si el gancho del acromion impedía la rotación externa debería ser removido. Sever también mencionaba desde entonces como secuela de este procedimiento la importante pérdida de la rotación interna por acción del músculo subescapular. ${ }^{10}$

L'Episcopo realizaba en 1934 la transferencia del redondo mayor, reinsertándolo en la dirección opuesta. ${ }^{11}$ Hoffer, Roper y Wickenden expusieron en 1978 la técnica de liberación capsular anterior, sección del pectoral mayor y transferencia de los músculos redondo mayor y dorsal ancho en conjunto para dar rotación externa al manguito rotador. ${ }^{12}$

\section{EXPLICACIÓN BIOMECÁNICA DE LA DEFORMIDAD}

La displasia glenohumeral ha sido motivo de estudio y aún existen interrogantes acerca de la patogénesis de la deformidad. Frost describe en 1997 el balance que debe existir entre la compresión y la tracción que genera la masa muscular sobre la cabeza humeral y que cualquier alteración en este balance excediendo el límite fisiológico inhibe el crecimiento óseo. ${ }^{13,14}$ Por otra parte, la inervación intacta es uno de los factores más importantes para regular el crecimiento óseo, por lo que una disrupción en la inervación de la articulación es una causa de limitaciones en su crecimiento. ${ }^{5}$

La articulación del hombro es una unidad con mucha movilidad y se controla por músculos que están comandados por todas las raíces del plexo braquial.

La fisiopatología de esta deformidad se considera que ocurre por el desbalance muscular progresivo entre rotadores internos y externos que progresivamente produce y aumenta retroversión del húmero, con elongación subsecuente de los rotadores externos y acortamiento de los internos, alterando finalmente el equilibrio muscular. ${ }^{15,16}$ Por lo general este tipo de deformidades se dan en lesiones C5-C6 o C5-6-7. El músculo infraespinoso tiene menos potencial de recuperación, mientras que el subescapular lo hace mejor y de manera más rápida, por lo que ya no existe este equilibrio dinámico.

Las fases de la displasia de la glenoides fueron en un inicio descritas por Pearl y Edgerton, ${ }^{17}$ posteriormente Kon y colaboradores correlacionaron estas fases con la rotación externa pasiva que presentaba el pacien- 
Tabla 1: Correlación de la displasia glenoidea con la rotación interna.

\begin{tabular}{ccc} 
Etapa & Clasificación Pearl & Rotación externa pasiva \\
\hline 1 & Cabeza humeral concéntrica y reducida & 0 \\
2 & Aplanamiento glenoideo & $-10^{\circ}$ \\
3 & Glenoides bicóncava & $-10^{\circ}$ \\
4 & Pseudoglenoides & $-20^{\circ}$ \\
\hline
\end{tabular}

te. ${ }^{18} \mathrm{El}$ desbalance muscular prolongado lleva a un aplanamiento y retroversión de la cabeza humeral. La glenoides se vuelve bicóncava con una faceta posterior falsa, que con el tiempo evoluciona a una pseudoglenoides y ocurre una luxación posterior de la cabeza humeral. ${ }^{19}$ Se desarrollan además cambios adaptativos como sobrecrecimiento del acromion y la parte lateral de la clavícula, los ligamentos coracohumeral y coracoacromial se contraen y causan una restricción mayor en el movimiento hacia la rotación externa (Tabla 1).

Existe una ley conocida como de Hueter-Volkmann que menciona que menor estrés conlleva a mayor crecimiento y que un estrés excesivo conduce al retraso del crecimiento, por lo que en 2013 se decidió comprobar los posibles resultados de este principio midiendo el efecto de la subluxación de la cabeza humeral en el crecimiento escapular de niños con lesiones de plexo braquial de nacimiento. De acuerdo con esta ley, la subluxación debería resultar en una disminución del crecimiento de la longitud dorsal de la escápula por un aumento de la presión dorsal, y un aumento de la longitud ventral por disminución de la presión. El estudio concluyó que la subluxación conduce a una disminución del crecimiento de la longitud dorsal de la escápula. ${ }^{20}$

\section{EXPLORACIÓN FÍSICA Y EVALUACIÓN}

La exploración física detallada es de suma importancia, pero puede ser dificultosa en el paciente pediátrico. Con paciencia y método se logran evaluar con diferentes pruebas los movimientos activos, pasivos y las limitaciones, realizando una documentación detallada.

Existen varios sistemas de evaluación para complementar la exploración que pueden agregar confusión al panorama. Bae y su equipo realizaron un estudio para validar la utilidad de los sistemas más utilizados, concluyendo que las escalas de Mallet, Toronto y la escala de movimiento activo son los instrumentos más confiables en pacientes con lesiones del plexo braquial de nacimiento. ${ }^{21}$

Gharbaoui $^{15}$ valora los arcos de movimiento específicos entre el húmero y la escápula para correlacionar directamente la función con las limitaciones de la articulación glenohumeral. Mide cinco ángulos particulares y esta escala se convierte en una forma muy precisa de documentar el proceso de 
restricción o el progreso de la recuperación durante el seguimiento de los pacientes.

1. Ángulo inferior: ángulo escapulohumeral en abducción (valor de 160 a 180). Se mide el ángulo entre el borde lateral de la escápula y el húmero llevado a la máxima abducción, estabilizando firmemente la escápula al tórax. Un ángulo menor indica contractura de la porción inferior de la articulación glenohumeral.

2. Ángulo superior: ángulo escapulohumeral en aducción (valor de 30 a 40). Se mide el ángulo entre el borde lateral de la escápula y el húmero con el brazo llevado a máxima aducción. Un ángulo mayor indica contractura de la parte superior de la articulación glenohumeral.

3. Ángulo posterior: ángulo escapulohumeral en aducción horizontal (valor de 90 a $\left.100^{\circ}\right)$. Con flexión a $90^{\circ}$ del hombro y abducción máxima se mide el ángulo entre el húmero y el eje de la escápula. Un ángulo mayor indica contractura posterior de la articulación glenohumeral.

4. Movimiento glenohumeral en rotación interna: se mide empezando con el húmero a $90^{\circ}$ de abducción y el codo a $90^{\circ}$ de flexión, estabilizando firmemente la escápula contra el tórax. Se lleva el brazo a rotación interna. Valor normal de 80 a $90^{\circ}$.

5. Movimiento glenohumeral en rotación externa: se empieza lo mismo que el anterior, pero llevando el brazo a rotación externa en el mismo plano. Valor normal de 75 a $90^{\circ}$ (Figura 1).

\section{CLASIFICACIÓN}

La primera clasificación para estas secuelas se realizó en 1981, clasificándolas en contracturas y en pacientes sólo con parálisis flácida.

En 1998 Waters hizo una clasificación radiológica demostrando adicionalmente que la deformidad es progresiva y aumenta con la edad (Tabla 2).

Waters en $1998^{15}$ establece una clasificación radiológica de la displasia glenohumeral que cuantifica el porcentaje de traslación de la cabeza humeral, su contorno así como la forma de la glenoides y su angulación.
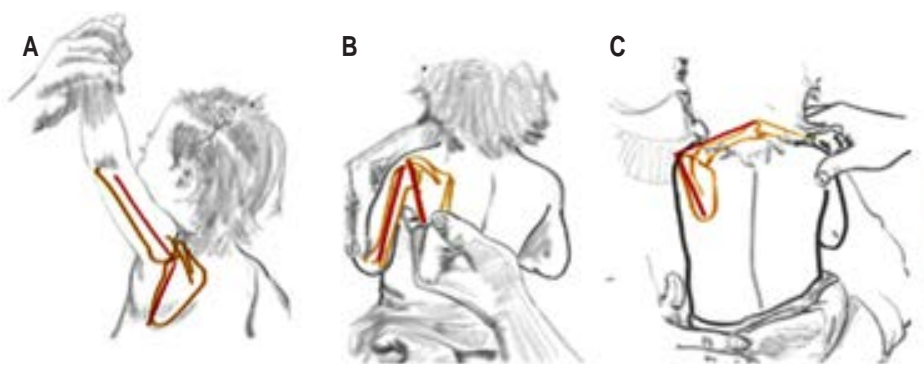

D

Figura 1: A) Ángulo inferior abducción. B) Ángulo superior aducción. C) Ángulo posterior. D) Movimiento glenohumeral en rotación externa.

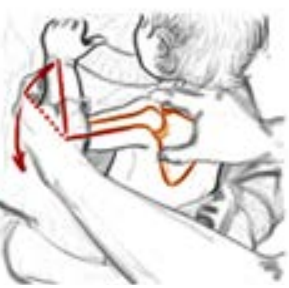




\section{Tabla 2: Clasificación de Waters.}

\begin{tabular}{cl} 
Grado & Hallazgos \\
\hline I & Glenoides normal: $<5^{\circ}$ de diferencia en retroversión en comparación con el lado contralateral normal \\
II & Deformidad mínima: $>5^{\circ}$ de diferencia en retroversión y sin subluxación posterior de la cabeza humeral \\
III & Moderada: subluxación posterior $<35 \%$ \\
IV & Severa: formación de pseudoglenoides \\
V & Aplanamiento severo de la cabeza humeral y glenoides con dislocación posterior progresiva o completa \\
& de la cabeza humeral \\
VI & Luxación articular glenohumeral en la infancia \\
VII & Arresto fisiario del húmero proximal
\end{tabular}

Al-Qattan ${ }^{22}$ en 2003 propuso una clasificación complementando la propuesta por Birch (Tablas 3 y 4) en 2001, incluyendo el resto de las deformidades como escápula alada y otras contracturas.

\section{IMAGENOLOGÍA}

El ultrasonido permite observar y cuantificar con exactitud la subluxación de la cabeza humeral. ${ }^{23,24}$ Se reporta una precisión de $82 \%$ para identificar estas lesiones. ${ }^{25}$

La resonancia magnética permite una evaluación más exacta, incluyendo la valoración del cartílago, definición de las deformidades de la cabeza humeral y la glenoides y caracterización de la atrofia muscular; sin embargo, requiere sedación en niños. ${ }^{15,26}$

La tomografía se utiliza en pacientes con madurez esquelética para una evaluación exacta de la deformidad ósea de la glenoides, cabeza humeral, acromion y coracoides. ${ }^{27}$

\section{TRATAMIENTO}

El tratamiento de esta secuela hoy en día es controversial debido a las múltiples técnicas quirúrgicas existentes y las estructuras que deben tomarse en cuenta para intervenir. En general se puede alcanzar una mejoría en la función, aunque nunca un retorno a la completa normalidad.

Los objetivos son reducir la articulación glenohumeral y reequilibrar el hombro para promover la remodelación y restaurar la función. ${ }^{28}$

Los principios fundamentales son liberar las estructuras anteriores (ligamento coracohumeral, subescapular, pectoral menor), asegurar una reducción concéntrica y restaurar la potencia de la rotación externa. ${ }^{6}$

Puede realizarse una liberación anterior abierta o artroscópica, la cual también muestra adecuados resultados, ya que esta última produce menos cicatriz y una adecuada visualización de toda la articulación. ${ }^{29}$

Siempre deberán valorarse las estructuras que se liberarán y generalmente se toman muchas decisiones de manera intraoperatoria. 
Tabla 3: Clasificación propuesta por Birch.

Tipo

Patología

I. Contractura en rotación interna con articulación congruente

Contractura del subescapular (SE)

II. Subluxación posterior simple

Lo anterior + cabeza humeral sobre pseudoglenoides

III. Luxación posterior simple

IV. Subluxación posterior compleja

Contractura del SE-cabeza humeral posterior a la glenoides

V. Luxación posterior compleja

Tipo II + elongación de la coracoides y acromion

Tipo IV + retroversión variable de la cabeza humeral

\section{BALANCE DE TEJIDOS BLANDOS}

Múltiples estudios han demostrado que restaurar la rotación externa ayuda a mejorar la función de la extremidad y corregir la displasia en hombros con capacidad de remodelación. ${ }^{30,31}$

El músculo más utilizado para restaurar la rotación externa es el dorsal ancho, en ocasiones con el redondo mayor, insertándolos en la tuberosidad mayor contrarrestando así la fuerza de rotación interna del subescapular y deprimiendo la cabeza humeral de manera similar a la función del supraespinoso. ${ }^{6}$

Pueden existir casos con contracturas residuales en abducción o rotación externa. Aquí la deformidad glenohumeral es infrecuente y puede asociarse a una escápula alada al intentar la rotación interna, pero por lo general su función es aceptable. En ocasiones se requiere una osteotomía desrotadora para corrección.

\section{PROCEDIMIENTOS SOBRE HUESO}

Se han descrito varias técnicas quirúrgicas para tratar la displasia, generalmente en niños mayores cuando ocurre una deformidad ya establecida.

La retroversión humeral puede corregirse con una osteotomía desrotadora en casos con más de 40 grados de retroversión. ${ }^{19} \mathrm{Al}$ corregirla mejora la posi-

Tabla 4: Clasificación propuesta por AI-Qattan.

\begin{tabular}{|c|c|c|}
\hline Tipo & Subtipo & Patología \\
\hline A. Parálisis global & & Parálisis de todos los músculos \\
\hline B. Parálisis de un grupo muscular & $\begin{array}{l}\text { I. Parálisis de rotadores externos } \\
\text { II. Parálisis de rotadores internos }\end{array}$ & $\begin{array}{l}\text { I. Parálisis de rotadores externos sin } \\
\text { contractura en rotación interna } \\
\text { II. Parálisis de rotadores internos sin } \\
\text { contractura en rotación externa }\end{array}$ \\
\hline C. Contractura en rotación interna & Birch I-V & Birch I-V \\
\hline D. Otras contracturas & $\begin{array}{l}\text { I. Contractura en rotación externa } \\
\text { II. Contractura en abducción }\end{array}$ & $\begin{array}{l}\text { I. Contractura de rotadores externos con } \\
\text { o sin subluxación anterior } \\
\text { II. Contractura del supraespinoso }\end{array}$ \\
\hline E. Escápula alada & & Parálisis del serrato anterior \\
\hline
\end{tabular}


ción de la articulación glenohumeral, pero puede generar una pérdida importante de la rotación interna. Debe realizarse a nivel de la inserción del deltoides y en casos con una displasia ya establecida. Muchos autores recomiendan efectuarla por vía anterior. ${ }^{32,33}$

Para el manejo de la displasia glenoidea y corregir la subluxación de la cabeza, tradicionalmente se aumentaba el labio posterior de la glenoides con injerto óseo, pero sus malos resultados se deben a que este manejo no restaura la congruencia articular perdida y no provee una superficie cartilaginosa, por lo que no se evita la evolución a una artrosis. Nuestra experiencia y la escasa evidencia en la literatura es que para que este procedimiento sea efectivo debe combinarse con procedimientos sobre los tejidos blandos y transferencias tendinosas. ${ }^{34}$

Mascio describió en $2011^{35}$ buenos resultados en 29 pacientes a los cuales les aplicó esta técnica logrando adecuada estabilidad glenohumeral y mejorando el puntaje en la escala de Mallet.

La artrodesis de hombro se utiliza poco y se realiza sólo en pacientes esqueléticamente maduros, con músculos trapecio, elevador de la escápula, serrato anterior y romboides preservados. Se reserva para lesiones totales, o pacientes con un hombro doloroso y por definición es un procedimiento que limita severamente el movimiento del hombro.

Métodos de tratamiento utilizados en el Diplomado de Plexo Braquial y Nervio Periférico con sede en el Hospital Universitario «Dr. Joaquín Del Valle Sánchez», Torreón, Coahuila (Tabla 5).

\section{4-11 meses}

En pacientes menores de un año el tratamiento siempre deberá basarse en evitar la evolución de la contractura y la luxación glenohumeral. Esto debe basarse

Tabla 5. Método de tratamiento propuesto por los autores.

\begin{tabular}{|c|c|c|c|c|}
\hline 4-11 meses & 1-4 años & 4-10 años & $>10$ a 25 años & > 25 años \\
\hline $\begin{array}{l}\text { - Terapia física } \\
\text { - Férula nocturna } \\
\text { - Aplicación de } \\
\text { bótox a músculos } \\
\text { con contractura } \\
\text { - Cirugía } \\
\text { a) Reconstrucción } \\
\text { nerviosa } \\
\text { b) Transferencia } \\
\text { nerviosa } \\
\text { c) Liberación de } \\
\text { contractura }\end{array}$ & $\begin{array}{l}\text { - Terapia } \\
\text { - Liberación de } \\
\text { contractura } \\
\text { a) Abierta } \\
\text { b) Artroscópica } \\
\text { - Transferencia } \\
\text { nerviosa selectiva } \\
\text { - Transferencia } \\
\text { muscular }\end{array}$ & $\begin{array}{l}\text { - Osteotomía desro- } \\
\text { tadora de húmero } \\
\text { - Osteotomía de } \\
\text { glenoides } \\
\text { - Osteotomía de es- } \\
\text { pina de la escápula } \\
\text { - Osteotomía de } \\
\text { acromion } \\
\text { - Transferencias } \\
\text { musculares } \\
\text { - Transferencias ner- } \\
\text { viosas selectivas } \\
\text { - Terapia física y } \\
\text { ocupacional }\end{array}$ & $\begin{array}{l}\text { - Osteotomía desrota- } \\
\text { dora de húmero } \\
\text { - Osteotomía de } \\
\text { glenoides } \\
\text { - Osteotomía de espi- } \\
\text { na de la escápula } \\
\text { - Osteotomía de } \\
\text { acromion } \\
\text { - Transferencias } \\
\text { musculares } \\
\text { - Transferencias } \\
\text { nerviosas selectivas } \\
\text { - Terapia física y } \\
\text { ocupacional }\end{array}$ & $\begin{array}{l}\text { - Prótesis reversa si } \\
\text { hay adecuada fun- } \\
\text { ción del deltoides } \\
\text { - Terapia física y } \\
\text { ocupacional }\end{array}$ \\
\hline
\end{tabular}


desde los primeros meses en la terapia física y programa de ejercicios en casa. Férula nocturna para evitar también lo mencionado anteriormente. La aplicación del bótox en casos de contracturas más severas también se utiliza junto con la terapia y la férula.

Si el paciente será sometido a cirugía de reconstrucción nerviosa y el grado de contractura es importante se prefiere realizar la liberación anterior de las estructuras (ligamento coracohumeral, subescapular, tendón del pectoral), con colocación posterior de férula en rotación externa durante cuatro semanas y posteriormente nocturna.

\section{1-4 años}

Después del año de edad, muchas veces es necesario realizar una liberación anterior y una reducción, ya que el paciente aún tiene capacidad de remodelación. Puede hacerse abierta o artroscópica. Además, se evalúa si realizamos una transferencia nerviosa selectiva, por ejemplo, para la rotación externa o una transferencia muscular simple o combinada para mejorar la rotación externa y/o abducción.

Terapia física siempre después de la cirugía.

\section{4-10 años y > 10 años}

En estos dos últimos grupos de pacientes que ya tienen una secuela establecida, el número de procedimientos quirúrgicos se vuelve mayor; el manejo debe ser individualizado después de una evaluación minuciosa de cada paciente, complementado con estudios imagenológicos para una adecuada planeación. Múltiples osteotomías de acuerdo al tipo de deformidad y transferencias musculares para mejorar la función. Las transferencias nerviosas selectivas se reservan para ciertos pacientes.

En los últimos años se ha utilizado la prótesis reversa como opción de salvataje en pacientes con una función adecuada del deltoides y que ya hayan desarrollado una displasia.

\section{CONCLUSIONES}

Las secuelas de hombro en el paciente con lesión de plexo braquial de nacimiento son motivo de estudio y un reto para el cirujano. Se requiere la identificación precoz y correcta de la causa de cualquiera de estas condiciones para limitar lo más posible las secuelas y lograr minimizar el impacto negativo que tienen dentro de la vida diaria del niño.

Se cuenta con múltiples modalidades de tratamiento y no existe un algoritmo a seguir, ya que el manejo debe ser individualizado para cada paciente.

Debido al inesperado hallazgo de esta patología y lo dificultoso que es tratarla, se requiere de un enfoque multidisciplinario y un manejo individualizado para mejorar la función y prevenir deformidades futuras en estos pacientes. 


\section{BIBLIOGRAFÍA}

1. Levine MG, Holroyde J, Woods JR Jr, Siddiqi TA, Scott M, Miodovnik M. Birth trauma: incidence and predisposing factors. Obstet Gynecol. 1984; 63 (6): 792-795.

2. Hardy AE. Birth injuries of the brachial plexus: incidence and prognosis. J Bone Joint Surg Br. 1981; 63-B (1): 98-101.

3. Michelow BJ, Clarke HM, Curtis CG, Zuker RM, Seifu Y, Andrews DF. The natural history of obstetrical brachial plexus palsy. Plast Reconstr Surg. 1994; 93 (4): 675-680; discussion 681.

4. Diagnóstico y tratamiento de la lesión obstétrica del plexo en niños y niñas de 0 a 15 años en el $2^{\circ}$ nivel de atención. México: Secretaría de Salud; 2012.

5. Hoeksma AF, Ter Steeg AM, Dijkstra P, Nelissen RG, Beelen A, de Jong BA. Shoulder contracture and osseous deformity in obstetrical brachial plexus injuries. J Bone Joint Surg Am. 2003; 85 (2): 316-322.

6. Nixon M, Trail I. Management of shoulder problems following obstetric brachial plexus injury. Shoulder Elbow. 2014; 6 (1): 12-17.

7. Birch R. Medial rotation contracture, posterior dislocation of the shoulder. In: Gilbert A (Ed). Brachial plexus injuries. Hampshire (UK): Taylor \& Francis; 2001. pp. 249-259.

8. Whitman R. VIII. The treatment of congenital and acquired luxations at the shoulder in childhood. Ann Surg. 1905; 42 (1): 110-115. doi: 10.1097/00000658-190507000-00008.

9. Fairbanks HT. A lecture on birth palsy: subluxation of the shoulder joint in infants and young children. Lancet. 1913; 1: 217-223.

10. Sever J W. Obstetric paralysis: report of eleven hundred cases. JAMA. 1925; 85: 1862-1865.

11. L'Episcopo JB. Tendon transplantation in obstetrical paralysis. Am J Surg. 1934; 25: 122-125. doi: 10.1016/S0002-9610(34)90143-4.

12. Hoffer MM, Wickenden R, Roper B. Brachial plexus birth palsies. Results of tendon transfers to the rotator cuff. J Bone Joint Surg Am. 1978; 60 (5): 691-695.

13. Frost HM. Biomechanical control of knee alignment: some insights from a new paradigm. Clin Orthop Relat Res. 1997; (335): 335-342.

14. Gkiatas I, Kostas-Agnantis I, Agathopoulos S, Papadopoulos D, Vekris M, Gelalis I, et al. The effect of peripheral nervous system in growing bone biomechanics. An experimental study. J Orthop. 2019; 16 (3): 289-292.

15. Gharbaoui IS, Gogola GR, Aaron DH, Kozin SH. Perspectives on glenohumeral joint contractures and shoulder dysfunction in children with perinatal brachial plexus palsy. J Hand Ther. 2015; 28 (2): 176183; quiz 184.

16. Waters PM, Smith GR, Jaramillo D. Glenohumeral deformity secondary to brachial plexus birth palsy. J Bone Joint Surg Am. 1998; 80 (5): 668-677.

17. Pearl ML, Edgerton BW. Glenoid deformity secondary to brachial plexus birth palsy. J Bone Joint Surg Am. 1998; 80 (5): 659-667.

18. Kon DS, Darakjian AB, Pearl ML, Kosco AE. Glenohumeral deformity in children with internal rotation contractures secondary to brachial plexus birth palsy: intraoperative arthrographic classification. Radiology. 2004; 231 (3): 791-795.

19. Kambhampati SB, Birch R, Cobiella C, Chen L. Posterior subluxation and dislocation of the shoulder in obstetric brachial plexus palsy. J Bone Joint Surg Br. 2006; 88 (2): 213-219.

20. van Gelein Vitringa VM, van Royen BJ, van der Sluijs JA. Scapular deformity in obstetric brachial plexus palsy and the Hueter-Volkmann law; a retrospective study. BMC Musculoskelet Disord. 2013; 14: 107.

21. Bae DS, Waters PM, Zurakowski D. Reliability of three classification systems measuring active motion in brachial plexus birth palsy. J Bone Joint Surg Am. 2003; 85 (9): 1733-1438.

22. Al-Qattan MM. Classification of secondary shoulder deformities in obstetric brachial plexus palsy. J Hand Surg Br. 2003; 28 (5): 483-486.

23. Pöyhiä TH, Lamminen AE, Peltonen JI, Kirjavainen MO, Willamo PJ, Nietosvaara Y. Brachial plexus birth injury: us screening for glenohumeral joint instability. Radiology. 2010; 254 (1): 253-260.

24. Vathana T, Rust S, Mills J, Wilkes D, Browne R, Carter PR, et al. Intraobserver and interobserver reliability of two ultrasound measures of humeral head position in infants with neonatal brachial plexus palsy. J Bone Joint Surg Am. 2007; 89 (8): 1710-1715.

25. Saifuddin A, Heffernan G, Birch R. Ultrasound diagnosis of shoulder congruity in chronic obstetric brachial plexus palsy. J Bone Joint Surg Br. 2002; 84 (1): 100-103.

26. van Gelein Vitringa VM, van Kooten EO, Mullender MG, van Doorn-Loogman MH, van der Sluijs JA. An MRI study on the relations between muscle atrophy, shoulder function and glenohumeral 
deformity in shoulders of children with obstetric brachial plexus injury. J Brachial Plex Peripher Nerve Inj. 2009; 4: 5.

27. Bhardwaj P, Burgess T, Sabapathy SR, Venkataramani H, Ilayaraja V. Correlation between clinical findings and CT scan parameters for shoulder deformities in birth brachial plexus palsy. J Hand Surg Am. 2013; 38 (8): 1557-1566.

28. Hui JH, Torode IP. Changing glenoid version after open reduction of shoulders in children with obstetric brachial plexus palsy. J Pediatr Orthop. 2003; 23 (1): 109-113.

29. Pearl ML, Edgerton BW, Kazimiroff PA, Burchette RJ, Wong K. Arthroscopic release and latissimus dorsi transfer for shoulder internal rotation contractures and glenohumeral deformity secondary to brachial plexus birth palsy. J Bone Joint Surg Am. 2006; 88 (3): 564-574.

30. Waters PM, Bae DS. The early effects of tendon transfers and open capsulorrhaphy on glenohumeral deformity in brachial plexus birth palsy. J Bone Joint Surg Am. 2008; 90 (10): 2171-2179.

31. Cohen G, Rampal V, Aubart-Cohen F, Seringe R, Wicart P. Brachial plexus birth palsy shoulder deformity treatment using subscapularis release combined to tendons transfer. Orthop Traumatol Surg Res. 2010; 96 (4): 334-339.

32. Waters PM, Bae DS. The effect of derotational humeral osteotomy on global shoulder function in brachial plexus birth palsy. J Bone Joint Surg Am. 2006; 88 (5): 1035-1042.

33. Julka A, Vander Have KL. Shoulder sequelae of neonatal brachial plexus injuries: orthopedic assessment and management. J Pediatr Rehabil Med. 2011; 4 (2): 131-140.

34. Dodwell E, O'Callaghan J, Anthony A, Jellicoe P, Shah M, Curtis C, et al. Combined glenoid anteversion osteotomy and tendon transfers for brachial plexus birth palsy: early outcomes. J Bone Joint Surg Am. 2012; 94 (23): 2145-2152.

35. Di Mascio L, Chin KF, Fox M, Sinisi M. Glenoplasty for complex shoulder subluxation and dislocation in children with obstetric brachial plexus palsy. J Bone Joint Surg Br. 2011; 93 (1): 102-107.

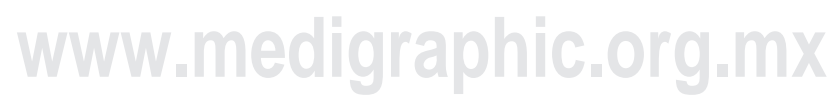

